\title{
L'HOMME L'Homme
}

Revue française d'anthropologie

217 | 2016

Varia

\section{Costumes et ethnicité}

À propos de Bernard Formoso, Costumes du Yunnan (Chine). Identité et symbolique de la parure, Nanterre, Société d'ethnologie, 2013

(« Ethnographie illustrée» 1).

Catherine Capdeville-Zeng

\section{OpenEdition}

\section{Journals}

Édition électronique

URL : http://journals.openedition.org/lhomme/28885

DOI : $10.4000 /$ lhomme.28885

ISSN : 1953-8103

Éditeur

Éditions de l'EHESS

Édition imprimée

Date de publication : 25 février 2016

Pagination : 123-132

ISSN : 0439-4216

Référence électronique

Catherine Capdeville-Zeng, "Costumes et ethnicité », L'Homme [En ligne], 217 | 2016, mis en ligne le 24 février 2018, consulté le 21 avril 2019. URL : http://journals.openedition.org/lhomme/28885; DOI :

10.4000/lhomme.28885

(c) École des hautes études en sciences sociales 


\title{
Costumes et ethnicité
}

\author{
Catherine Capdeville-Zeng
}

$C_{\mathrm{B}}$

MINCE ouvrage illustré de belles photographies propose des études des costumes de huit "nationalités " minzu minoritaires (shaoshu minzu), parmi les vingt-six que compte la mosaïque ethnique de la province du Yunnan, en République populaire de Chine. Ces costumes de minorités sont des "manifestes identitaires silencieux", à la fois "signes de reconnaissance et marques de distinction"(p. 12). Pour les membres d'un groupe, ils permettent d'identifier immédiatement une personne "fréquentable, apparentée ou épousable» (p. 13). Pour les personnes extérieures, ils permettent de situer leurs porteurs dans une ethnie localement reconnue. Arborés à l'occasion des fêtes ou des marchés, et non dans l'intimité des communautés villageoises, ou alors en version simplifiée, ces costumes "ancrent une personne dans le jeu des assignations identitaires"(id.). Ce sont les femmes qui portent les vêtements d'apparats les plus élaborés, et ce sont elles qui les confectionnent avec beaucoup de patience et de soin, selon des canons de beauté propres à leur groupe.

L'intérêt du livre est d'aller au-delà des critères esthétiques pour démontrer que les costumes sont des vecteurs de messages symboliques. Ils traduisent à la fois des aspects rituels fondamentaux, notamment ils sont "protecteurs" (p. 14) et "prophylactiques» (p. 15), et des idéesvaleurs importantes de ces sociétés. À l'image de l'homme qui a beaucoup travaillé pour créer un environnement et un paysage agricole dont il tire 
sa subsistance - par exemple, avec les rizières étagées construites par plusieurs minorités sur les pentes abruptes des vallées -, les costumes doivent évoquer les idées d'ordonnancement et de complétude du monde et des relations sociales. En outre, ces costumes « ne sont nullement la manifestation pétrifiée de cultures en voie de disparition» (p. 16). Au contraire, ils évoluent constamment, montrant ainsi que les groupes minoritaires soumis à des processus d'acculturation ont les moyens de négocier "le maintien de la structure sémiotique de leurs costumes sous couvert de concessions formelles " (id.).

J'ai choisi d'examiner plus particulièrement ici trois des minorités présentées dans le livre, dont les cas m'ont paru les plus intéressants du point de vue des enjeux identitaires qu'elles représentent, des emprunts culturels mutuels qu'elles pratiquent, et des relations qu'elles entretiennent avec les Han majoritaires. Je détaillerai d'abord les costumes singuliers des Taï Sai, qui incorporent de nombreux éléments inspirés de la cosmologie chinoise, puis les nouveaux « costumes folkloriques » des Jingpo et des Bai, qui marquent leur identité nouvelle, tout en la folklorisant.

\section{Les costumes des Taï Sai}

Les Taï Sai ne comptent que quelque 18000 personnes, établies dans le fond de vallées dont la confluence aboutit au fleuve Rouge à Jiasa dans le centre du Yunnan, dans le district yi de Xinping. Ils se distinguent des autres Taï du Yunnan par leurs costumes, en partie empruntés aux Yi, et décorés de riches broderies, raison pour laquelle ils sont appelés par les Chinois les "Taï à la taille fleurie» (Hua Yao Dai). Assimilé aux Dai (Taï), leur petit groupe ne représente pas une "nationalité " minzu reconnue en Chine; leur système religieux est resté à l'écart du bouddhisme, mais, "sans doute par l'entremise de Yi, il a intégré certains éléments du symbolisme chinois» (p. 85).

Ce peuple illustre à merveille «l'étroite et constante interaction instaurée dans le monde chinois entre le microcosme corporel et le macrocosme englobant» (p. 87). Par exemple, le chapeau conique des femmes "figure les caractéristiques du paysage local et reproduit les dispositifs de protection contre les esprits maléfiques appliqués à la maison et au village» (id.). Le chapeau comprend une bordure de couleur orange (évoquant le fleuve Rouge) et un cône de couleur jaune (évoquant le mont Ailao). Bernard Formoso indique que, "par emprunt au symbolisme chinois, le jaune et le rouge sont auspicieux car ils véhiculent l'idée de bonheur et de richesse " (id.). Quant à la structure interne du chapeau, elle représente le ta lew, qui est un objet porte-bonheur et conjuratoire, 
fait de croisillons de bambous accrochés au-dessus des portes et fenêtres. Le ta lew protège donc aussi la tête des femmes, tout comme leurs mains sur lesquelles il est également tatoué. La partie supérieure du corps - la plus importante - est ainsi préservée des dangers extérieurs par ce que l'auteur appelle un "système de défense» (p. 89).

Par ailleurs, les motifs du chapeau sont regroupés selon un mode ternaire. Or, chez les Taï, le chiffre trois symbolise la vie; dans la cosmologie chinoise, il évoque la trinité stellaire - les trois étoiles de la bonne santé, de la prospérité et de la longévité -, exprimée par l'association des couleurs rouge, jaune et vert. Les Taï Sai se désignent comme les "gens des trois rivières ", celles qui garantissent la fertilité des rizières, mais aussi la fécondité des femmes "selon le principe des vases communicants entre macro et microcosmes et par emprunt possible aux idées maîtresses de la géomancie chinoise» (p. 93).

La coupe de la veste et du boléro, en forme de losange, est nommée par le terme chinois "papillon ». Cela peut surprendre puisque, dans le monde taï, les papillons sont les incarnations d'esprits maléfiques et, dès lors, vecteurs d'infortune. C'est pourquoi l'auteur émet l'hypothèse que si les Taï Sai, «sous l'influence des Yi ou des Han, ont adopté le terme chinois pour papillon ", c'est pour s'arroger «les idées auspicieuses qu'il véhicule. En effet, pour les Han comme pour les Yi, le papillon évoque l'innocence, la légèreté, la joie, l'été et le bonheur conjugal » (id.). Et d'en conclure que "les influences chinoises, opérant directement ou par l'entremise des Yi, sont manifestes chez les Taï Sai. Cependant l'acculturation procède ici, comme dans bien d'autres contextes culturels, d'une appropriation dynamique, facteur de singularité»(p. 96). Bernard Formoso laisse en effet entrevoir une relation pacifiée entre eux, les Taï Sai n'hésitant pas à emprunter les symboles chinois à leur convenance, soit parce qu'ils véhiculent des idées semblables aux leurs (le ternaire), soit justement parce qu'ils leur paraissent mieux adaptés que les leurs (le papillon).

\section{Les costumes des Jingpo}

Les Jingpo, parlant une langue sino-tibétaine, se répartissent entre le Myanmar (État Kachin) et le sud-ouest du Yunnan (Chine), et correspondent à une population d'environ 500000 individus. Ils sont connus par les travaux du père Charles Gilhodes (1922) et d'Edmund Leach (1954), qui décrivent une société longtemps divisée en "strates »: nobles, roturiers et esclaves. Depuis une trentaine d'années est apparu un "costume ethnique» (p. 30), une tenue d'apparat portée de façon identique des deux côtés de la frontière. En effet, la montée d'un ethno- 
nationalisme kachin en Birmanie mobilisa les leaders jingpo et les incita à nouer des contacts étroits avec les représentants de leur minorité en Chine, influençant ainsi la naissance d'un "pan-ethno-nationalisme » jingpo (p. 30). En cristallisant les premiers signes culturels d'appartenance, les costumes apparurent dès lors comme des marqueurs ethniques. L'essor du tourisme offrit par la suite "une tribune valorisante à cette coproduction essentialiste et folklorisée des coutumes» (p. 31). À l'occasion de la fête Munao, qui attire chaque année des centaines de milliers de touristes, les Jingpo portent ces costumes traditionnels pour afficher leur identité et défendre leur culture. Au point que cette fête emblématique "a été expurgée graduellement de ses facteurs négatifs et est devenue un grand festival des Jingpo, de même qu'une indication de leur nouvelle vie "heureuse" " 1 .

Le vêtement recomposé des Jingpo de Dehong symbolise donc leur «personnalité culturelle» (id.), notamment leur rapport à l'ancestralité et leurs aspirations fondamentales. Sans rentrer dans le détail de la riche comparaison menée par l'auteur entre le costume ancien et le "costume nouvelle manière» (p. 33), j'aimerais m'arrêter sur quelques nouveaux éléments dans l'agencement de ces costumes qui me paraissent essentiels.

On constate, d'abord, la transformation de l'encolure faite de plusieurs rangées de larges boutons en argent en une nouvelle collerette comportant trois rangées de demi-sphères en argent ou en cuivre. Ces demi-sphères représentent la lune et sont indissociables de plaques rondes du même métal, frappées d'une étoile et portées soit en collier, soit cousues au dos de la veste. L'étoile évoque, quant à elle :

«[...] le soleil rayonnant dans le ciel figuré par le rond. Se trouve ainsi exprimée une complémentarité qu'affichent très souvent les costumes de populations du monde chinois : celle de la lune et du soleil, avec pour corollaires aux différents niveaux de la cosmologie le principe femelle (yin) tel qu'il se combine au principe mâle (yang), et la femme telle qu'elle s'unit à l'homme» (id.).

On observe, ensuite, que les broderies de la jupe sont considérablement simplifiées par rapport à celles de jadis, qui nécessitaient des milliers d'heures de travail. Pour les informateurs, ce constat est révélateur du fait que les femmes sont davantage accaparées par les travaux agricoles depuis que les cultures sont devenues intensives, et n'ont donc désormais plus assez de temps pour confectionner ces broderies. Enfin, le costume jingpo moderne est plus coloré, la coiffe est décorée de motifs qui n'existaient pas autrefois et des pompons sont apparus sur la jupe et la coiffe.

1. Selon le site internet du gouvernement du Yunnan, cité par l'auteur (p. 31). 
Le festival du Munao se présente «comme une synthèse originale des Munao du temps jadis " et comme "reprenant des éléments des anciens Munao funéraires» (p. 39). Cependant, aujourd'hui ces fêtes se font «sans distinction statutaire au profit de tous les défunts " (id.) et «ne visent pas à dissocier le mort du monde des vivants. À l'inverse, elles placent les Jingpo dans la continuité de la geste de leurs ancêtres» (p. 41).

L'auteur termine par une présentation des parures des Jingpo de la vallée de la Salween, qui ont, pour leur part, conservé de nombreux motifs anciens, comparables aux descriptions qu'en a faites Charles Gilhodes au début du $\mathrm{XX}^{\mathrm{e}}$ siècle. Bernard Formoso explique cette particularité par le fait que les Jingpo de cette vallée sont peu nombreux et vivent dispersés, de sorte que :

«[...] leur marginalité les protège de la pression normative que leurs homologues de Dehong ont subie il y a des décennies en vue d'un affichage unitaire de leur spécificité culturelle. Vivant hors des circuits organisés par les opérateurs du tourisme et n’organisant pas de grands Munao, ils n'ont pas à jouer le jeu d'une mise en représentation folklorique d'eux-mêmes» (pp. 44-45).

À partir de ces descriptions de costumes, on perçoit donc l'ampleur de l'évolution du particularisme jingpo durant les trente dernières années: l'apparition du costume folklorique à visée identitaire en lien avec le succès touristique d'un grand festival traditionnel ; une identité cependant influencée par l'idéologie englobante chinoise; la transformation du travail des femmes et le nivellement des statuts sociaux; ainsi que la possibilité pour des populations vivant dans des zones plus retirées de mieux conserver leurs traditions.

\section{Les costumes des Bai}

Les quelque deux millions de Bai, installés près du lac Erhai dans la région de la ville de Dali au nord-ouest du Yunnan, parlent une langue de la famille sino-tibétaine, dont $70 \%$ du vocabulaire est emprunté au chinois. Ils fondèrent, au VIII ${ }^{\mathrm{e}}$ siècle, le royaume multi-ethnique de Nanzhao (737-902), auquel succéda, au Xe siècle, le royaume bai de Dali. En 1253, ce dernier fut renversé par l'armée mongole, avant de passer sous administration chinoise et être définitivement intégré au Yunnan (1274), qui demeura depuis cette date une province chinoise.

Les Bai sont bien connus grâce à la monographie que leur a consacrée Francis Hsu en 1948, Under the Ancestors' Shadow. Dans cet ouvrage écrit un an avant la révolution de 1949 et la proclamation de la République populaire de Chine, l'auteur ne distingue pas encore les Bai (appelés alors Min Chia ou Minjia en transcription pinyin) des Han majoritaires. 
Bernard Formoso évoque les débats suscités par les données de Francis $\mathrm{Hsu}$, notamment après que Claude Lévi-Strauss s'est appuyé sur l'un des articles de cet auteur (1945) dans les Structures élémentaires de la parenté (1949) pour prouver la prévalence du mariage croisé matrilatéral en Chine, à savoir $70 \%$ de tels mariages. Or, le choix de cette donnée a été critiqué par l'anthropologue américain Bernard Gallin (1963), pour qui elle ne peut s'appliquer aux Han.

Plus récemment, l'anthropologue chinois Liang Yongjia (2010) est revenu sur cette question. Il avance que la distinction entre Bai et Han aurait été en fait signifiée (c'est-à-dire "créée ?") après l'avènement de la République populaire de Chine en 1949, avec l'octroi du statut de "nationalité " minzu bai aux Minjia en 1956, qui se pensaient jusqu'alors semblables aux Han : «la différence entre Han et Minjia n'était ni importante à Dali, ni considérée comme une différence ethnique» (Ibid.: 78). Et d'ajouter :

«Je pense qu'il est des plus probable que Hsu et ses informateurs de West Town étaient tout à fait conscients des caractéristiques locales de West Town, mais aucun d'eux ne considérait ces caractéristiques locales comme ce que l'on appelle aujourd'hui des caractéristiques ethniques. Il en va ainsi parce que, en tout premier lieu, les "caractéristiques locales" sont un phénomène courant en Chine» (Ibid.: 81).

Les différentes lectures de Under the Ancestors' Shadow accusant Francis Hsu d'avoir commis une "erreur ethnique " parce qu'il décrivait les Min Chia comme étant semblables aux Han, pèchent donc par un manque de connaissance de la situation locale précédant la révolution communiste. Des auteurs tels que Edmund Leach (1950), Colin Mackerras (1988), Stevan Harell (1995), sans compter les spécialistes chinois et bai, ont tous critiqué Francis Hsu à ce sujet. Du côté de l'anthropologie française, on peut ajouter Laurent Barry $(2008)^{2}$. Quant à Jacques Lemoine, en raison des discussions sur les Bai, et notamment d'un colloque tenu en 1957 au Yunnan « où l'unanimité [entre linguistes et historiens] n'avait toujours pas pu se faire sur l'origine et la formation de cette ethnie atypique " (1978: 834), il qualifie les Bai d' "ethnie controversée " dans le sous-titre de l'article qu'il leur consacre dans Ethnologie régionale 2 de l'encyclopédie de la Pléiade (Ibid. : 2035).

2. «Un point est omis dans le commentaire de Lévi-Strauss : celui qui nous apprend que la population de la ville étudiée par Hsu (1945) [...] n'est pas Han mais d'origine Min Chia et qu'elle ne parle même pas le chinois (cf. Gallin 1963). Considérer alors que les pratiques Min Chia nous informeraient sur celles des Han, revient à peu près au même que de conduire une lecture du système de parenté chinois à partir de l'ethnographie des $\mathrm{Na}$ matrilinéaires qui ignorent la figure paternelle et l'institution du mariage, en arguant du fait qu'ils foulent eux aussi les terres de l’Empire du milieu» (Barry $2008:$ 713-714). 
Depuis 1956, comme le souligne Bernard Formoso, la «nationalité » bai s'est affirmée, tandis que Dali, dont les sites alentour ont été inscrits sur des listes du patrimoine naturel de l'Unesco et sur des listes nationales chinoises, est devenue aujourd'hui l'une des villes touristiques les plus célèbres du Yunnan et de Chine. Dès 1982, le gouvernement de la préfecture autonome bai de Dali a incité son peuple à concevoir et promouvoir - par exemple, en demandant aux travailleurs de l'industrie touristique de le porter -, un nouveau " costume ethnique ", plus coloré, "de nature selon lui à refléter la joie de vivre des Bai au sein de la République populaire, et à afficher leur adhésion à la politique d'ouverture et de réformes de la "nouvelle Chine" " (p. 65). Une version folklorisée de ce costume bai est vendue aux touristes, en même temps que des tissus folkloriques supposés "bai traditionnels" confectionnés par un artisanat local.

Aujourd'hui, ce nouveau costume multicolore est plutôt arboré par les femmes de moins de quarante ans. L'ancien, composé d'une sobre tunique bleue et d'un tablier noir remontant sur la poitrine richement ornementé dans sa partie inférieure, est toujours porté par les femmes plus âgées. Outre les couleurs, la différence la plus manifeste entre les deux se situe au niveau de la coiffe, dont l'auteur nous offre une comparaison détaillée sur plusieurs pages. Tout comme l'ancienne, la nouvelle coiffe s'inspire des éléments caractéristiques de la culture bai renvoyant au paysage local - la neige, les fleurs et le vent -, mais seulement en tant que références à des lieux aujourd'hui hautement touristiques de Dali : ainsi la couleur blanche évoque-t-elle les neiges éternelles du mont Cangshan, les «fleurs papillons " multicolores la très attractive Source aux papillons et les fleurs de lotus de la rangée inférieure les sites emblématiques (temples et pagodes) du bouddhisme ancien, tandis que le flot de fils blancs cousus à gauche figure le vent. Or, si la coiffe traditionnelle intégrait déjà une "allégorie du paysage", "celle-ci servait de moyen prophylactique au lieu d'être simplement figurative» (pp. 69-70). En outre, les coiffes traditionnelles différaient selon l'âge et le statut matrimonial des femmes qui les portaient, en sorte que :

"[...] l'évocation des sites remarquables de Dali, qui était secondaire dans la coiffe traditionnelle, est devenue le thème majeur de son avatar folklorisé. En contrepartie, celle-ci a perdu toute efficacité symbolique» (p. 73).

$\mathrm{La}$ " coiffe carte postale » qui a remplacé la " coiffe protectrice » est vendue aux touristes et sert "à signifier à des fins de propagande la "belle vie" du peuple bai dans la "nouvelle Chine" "(id.). Les motivations personnelles et familiales des femmes qui brodaient leurs coiffes sont complètement occultées au profit du nouveau modèle standardisé. 
Bernard Formoso termine sa présentation en émettant l'hypothèse que la sobriété vestimentaire des femmes bai allant de pair avec l'avancement en âge, " on peut supposer qu'elles seront plus enclines à renouer avec les tenues de leurs aînées qu’à porter des vêtements clinquants et incapables de satisfaire des angoisses existentielles qui, elles aussi, augmentent avec l'âge (santé, longévité, notamment)» (id.). J'aimerais être aussi optimiste que l'auteur... À l'inverse, sur mon terrain chinois du district de Nanfeng ${ }^{3}$ dans la province du Jiangxi, je vois aujourd'hui des femmes âgées abandonner la tunique bleue qui leur est réservée pour des tuniques fleuries et colorées qui sont normalement le privilège des jeunes femmes lors des fêtes des temples. Cela renvoie aussi, en arrière-plan, à la question universelle de l'émancipation des femmes : qu'elles soient han, bai (minjia) ou hakka, certaines femmes en Chine défendent leur droit de s'habiller comme elles l'entendent, sans plus devoir se soumettre à la coutume, à partir du moment où elles sont ménopausées, en portant des vêtements de couleurs tristes.

Parmi les trois sociétés présentées ici, la première, la société taï sai, est celle qui paraît avoir élaboré ses costumes de la façon la plus libre et paisible. Les emprunts stylistiques ne semblent pas, en effet, avoir été imposés par la domination des Han, comme tend par exemple à le démontrer l'usage du mode ternaire qui est tout autant un élément fort du symbolisme chinois que de celui des "gens des trois rivières". Chez les Jingpo, en revanche, la recherche d'une expression identitaire à la fois locale et transfrontalière apparaît comme plus affirmée, ce qui, en un sens, sous-entend l'existence d'une sorte de conflit non évoqué avec les Han voisins. Leur costume arbore désormais les caractères signifiants de leur spécificité culturelle au point de la folkloriser. Les valeurs traditionnelles véhiculées autrefois par l'intermédiaire de l'ancien costume s'en sont trouvées profondément transformées, sauf dans les régions reculées, où le tourisme n'a pas encore pénétré. Enfin les Bai, marqués par un réveil identitaire récent et une reconnaissance nouvelle de la part des Chinois, se sont créé un "costume carte postale» en rupture totale avec leurs costumes traditionnels, dont les aspects essentiels et prophylactiques ont été relégués au second plan, voire ignorés. Ce nouveau costume folklorique est significatif de la société bai moderne, où la valeur "beauté » est désormais exaltée à des fins touristiques, aux dépens des aspects rituels d'intercession et des idées d'ordonnancement sociocosmique du monde.

3. Région dont les habitants sont probablement, mais de façon non reconnue, d'origine hakka, un "peuple» han non inscrit dans la liste des «nationalités » officielles, mais dont le sentiment d'identité a fortement progressé dans certains lieux plus au sud de cette province. 
Cette société s'éloigne donc des principes traditionnels d'englobement du microcosme corporel par le macrocosme, pourtant au fondement de la pensée chinoise, comme l'a montré à plusieurs reprises Bernard Formoso. "L'ambition esthétique» (p. 105) supplante alors, tout en l'abolissant, la vocation première de ces costumes, qui était de porter bonheur et de protéger ceux qui les revêtaient.

Mais l'auteur veut conserver une vision optimiste des changements qu'il décrit dans la globalité des sociétés qu'il a étudiées, car ils " touchent surtout les matériaux utilisés et sont finalement peu importants. Les costumes conservent l'essentiel de leurs fonctions et les conditions de leur usage ont peu évolué» (p. 108). "Pour être indiscutables, ces changements restent malgré tout placés sous le sceau d'un rapport de forces qui favorise l'expression des cultures locales en dépit des tentatives d'uniformisation conduites en ce sens par l'État» (id.).

Il faudrait pourtant insister sur le fait que les situations de ces peuples sont à chaque fois fort différentes, et que certains ont bien plus modernisé et folklorisé leurs costumes que d'autres. À mes yeux, le costume bai est celui qui a le plus été dénaturé par la "globalisation touristique" parmi tous ceux étudiés ici, même si, selon les dires de l'auteur, il garde des caractères d'origine tels que la ceinture ventrale toujours porteuse de prospérité. Il symbolise en fait l'exemple le plus flagrant, dans ce livre, de perversions des coutumes induites par l'influence chinoise. Est-ce à dire que cette minorité, en obtenant sa reconnaissance avec le statut de "nationalité " minzu, a dû en même temps transformer ses pratiques rituelles, ses valeurs, qu'elle s'est acculturée à partir du moment où elle entrait dans le processus de folklorisation qu'elle s'est infligée à elle-même sous la pression des Han et de la modernisation du pays imposée à tous (Han y compris) ? Leur "véritable» identité maintenant reconnue, mais devant jouer le rôle du "bon sauvage " pour les touristes han et étrangers, les Bai n'apparaissent-ils pas encore plus "dominés" qu'autrefois? À moins que, pour ce peuple de "barbares cuits", selon la terminologie chinoise ancienne qui opposait les "barbares cuits " (shufan = acculturés) aux «barbares crus" (shengfan = ensauvagés), ce nouveau costume tel qu'il a été personnalisé ne soit l'expression de leur résistance à l'ingérence han?

Ainsi ce petit livre, au-delà des descriptions des costumes qu'il présente, propose une réflexion élargie sur les relations entre les peuples qui se côtoient dans cette province du Yunnan et sur la modernisation en cours en Chine. Il mérite tout à fait une lecture approfondie.

Institut national des langues et civilisations orientales ASIES - CECMC/CNRS, Paris catherine.capdeville@inalco.fr 
MOTS CLÉS/KEYWORDS : Chine/China - Yunnan - minorités ethniques/ethnic minorities costume - folklore - identitélidentity - cosmologie chinoise/chinese cosmology.

\section{Glossaire des termes chinois cités dans le texte}

$\begin{array}{lll}\text { Bai(zu) } & \text { 白族 } & \text { les Bai (nationalité bai) } \\ \text { Dai(zu) } & \text { 傣族 } & \text { les Dai (nationalité dai - les Taï) } \\ \text { Hua Yao Dai } & \text { 花腰傣 } & \text { les Hua Yao Dai (Taï à la taille fleurie Taï Sai) } \\ \text { Jingpo (zu) } & \text { 景颇族 } & \text { les Jingpo (nationalité jingpo) } \\ \text { Minjia } & \text { 民家 } & \text { les Minjia (Min Chia) } \\ \text { minzu } & \text { 民族 } & \text { peuple, ethnie, nationalité, nation } \\ \text { shengfan } & \text { 生番 } & \text { "barbares crus» (ensauvagés) } \\ \text { shufan } & \text { 熟番 } & \text { "barbares cuits " (acculturés aux rites chinois) } \\ \text { shaoshu minzu } & \text { 少数民族 } & \text { nationalité minoritaire (minorité nationale) }\end{array}$

\section{RÉFÉRENCES CITÉES}

Barry, Laurent

2008 La Parenté. Paris, Gallimard

(«Folio. Essais » 498).

Gallin, Bernard

1963 "Cousin Marriage in China ", Ethnology 2 (1) : 104-108.

\section{Gilhodes, Charles}

1922 The Kachins. Religion and Customs. Bangkok, White Lotus.

\section{Harrell, Stevan}

1995 "Civilizing Projects and the Reaction to Them ", in Stevan Harrell, ed., Cultural Encounters on China's Ethnic Frontiers. Seattle-London, University of Washington Press : 3-36.

\section{Hsu, Francis}

1945 "Observations on Cross-Cousin Marriage in China ", American Anthropologist new ser. 47 : 83-103.

1948 Under the Ancestors' Shadow.

Chinese Culture and Personality.

New York, Columbia University Press.

\section{Leach, Edmund}

1950 "Review of Under the Ancestor's Shadow. Chinese Culture and Personality by Francis K. Hsu [43] ", Man 50 : 36.
1954 Political Systems of Highland Burma. A Study of Kachin Social Structure. London, G. Bell and Sons.

\section{Lemoine, Jacques}

1978 «Les Paï : une ethnie controversée, son identification liée à celle des populations de l'ancien royaume polyethnique de Nam Tchao - répartition - l'agriculture, l'élevage - traits culturels", in Jean Poirier, ed., Ethnologie régionale, 2. Asie, Amérique, Mascareignes. Paris, Gallimard («Encyclopédie de la Pléiade ») : 832-834 et 2035 [table analytique].

\section{Lévi-Strauss, Claude}

1949 Les Structures élémentaires de la parenté. Paris, Presses universitaires de France.

\section{Liang, Yongjia}

2010 " "The Ethnic Error" in Under the Ancestors Shadow and Dali Society in the Period of the Nationalist Governement ", Chinese Sociology and Anthropology 420 (4): 78-94.

\section{Mackerras, Colin}

1988 "Aspects of Bai Culture: Change and Continuity in a Yunnan Nationality", Modern China 14 (1) : 51-84. 